\title{
Impact of Upward Lymph Node Dissection on Survival Rates in Advanced Lower Rectal Carcinoma
}

\author{
Keisuke Uehara Seiichiro Yamamoto Shin Fujita Takayuki Akasu \\ Yoshihiro Moriya \\ Division of Colorectal Surgery, National Cancer Center Hospital, Tokyo, Japan
}

\section{Key Words}

Lymph node dissection - Upward lymph node dissection • Lower rectal carcinoma

\begin{abstract}
Background/Aims: This study investigated appropriate level of upward lymph node (LN) dissection in advanced lower rectal carcinoma. Methods: A total of 285 consecutive patients with stage II/III lower rectal carcinoma were analyzed. LN dissection was classified as follows: division of the root of the superior rectal artery (UD2), division of the root of the inferior mesenteric artery (UD3) and UD3 with para-aortic LN dissection (UD4). Results: LN metastases at the root of the inferior mesenteric artery were found in 4 patients. Their prognoses were worse than those of the other stage III patients $(p=0.011)$. On the other hand, LN metastases along the superior rectal artery were discovered in 14 patients, whose 5 -year overall survival rate was $61.2 \%$. By removing the LNs either UD2 or UD3/4, a similar survival rate was achieved in stage III patients with LN metastases along the superior rectal artery. Conclusion: Survival of a minority with metastatic LNs at the root of the inferior mesenteric artery was poor. Additionally, survival is no worse in patients with positive LN along the superior rectal artery as long as these positive nodes are resected by either UD2 or UD3/4. Low ligation is adequate for advanced lower rectal carcinoma.

Copyright $\odot 2007$ S. Karger AG, Basel
\end{abstract}

\section{Introduction}

It is well known that lower rectal carcinoma has two routes of lymphatic spread, i.e. upward and lateral spread. There have been many reports that discuss the significance of lateral pelvic lymph node dissection for advanced lower rectal carcinoma [1-4]. However, there have not been any definitive conclusions and various opinions have been expressed around the world. On the other hand, the impact of upward lymph node dissection for sigmoid colon or upper rectal carcinoma has been discussed in several reports [5-7], and yet few studies have focused on this issue in advanced lower rectal carcinoma. Although Pezim et al. [8] reported that high ligation of the inferior mesenteric artery had no survival advantage for rectal carcinoma patients, no counterarguments have been published and it remains difficult to generalize about the impact of upward lymph node dissection. The appropriate extent of upward lymph node dissection for advanced lower rectal carcinoma remains an unsolved issue and guidelines need to be established.

This study presents a detailed estimation of how the level of upward lymph node dissection affects survival rates following curative resection in advanced lower rectal carcinoma.

\section{KARGER}

Fax +4161306 1234 E-Mail karger@karger.ch www.karger.com
(C) 2007 S. Karger AG, Basel

0253-4886/07/0245-0375\$23.50/0

Accessible online at:

www.karger.com/dsu
Seiichiro Yamamoto

Division of Colorectal Surgery, National Cancer Center Hospital

5-1-1, Tsukiji, Chuo-ku

Tokyo 104-0045 (Japan)

Tel. +81 33542 2511, Fax +8133542 3815, E-Mail seyamamo@ncc.go.jp 


\section{Patients and Methods}

Between 1990 and 2002, a series of 303 consecutive patients at the National Cancer Center Hospital, Tokyo, underwent curative surgery for stage II or III lower rectal carcinoma. Lower rectal carcinoma was defined as a tumor with a distal margin $7 \mathrm{~cm}$ or less from the dentate line by digital examination and/or proctoscopy. Five patients with a history of malignancy (sigmoid colon carcinoma in 3 and bladder carcinoma in 2), who previously underwent lymph node dissection along the inferior mesenteric artery or in the lateral pelvis, were excluded, because the routes of lymphatic spread seemed to be changed in these cases. Two patients with synchronous advanced rectosigmoid carcinoma were excluded. Three stage II patients and 8 stage III patients did not undergo lymph node dissection along the inferior mesenteric artery but only in the mesorectum (UD1), because of preoperative underestimation. These 11 patients were also excluded. Consequently, 285 patients were eligible for this study. The mean (SD) distance from the dentate line of the tumor was 2.4 (1.0) (range $0.0-7.0) \mathrm{cm}$. No patients received preoperative radiotherapy and/ or chemotherapy. All patients were evaluated before surgery by total colonoscopy, barium enema and computed tomography. To evaluate comorbid conditions, cardiopulmonary function and renal function tests were performed. In our study, lateral pelvic lymph nodes were regarded as regional lymph nodes according to the Japanese classification of colorectal carcinoma [9], although lateral pelvic lymph node metastases are regarded as distant metastases in the TNM classification system [10]. Clinical stage II or III middle or lower rectal carcinoma, located at or below the peritoneal reflection, is an indication for lateral pelvic lymph node dissection in our hospital $[2,3]$. Postoperative adjuvant chemotherapy using oral or intravenous fluoropyrimidines was administered for 6 months to 27 stage III patients. Two stage III patients received postoperative radiotherapy and another underwent concomitant chemoradiotherapy.

The incidence of upward lymph node metastases based on histopathological data from the resected specimen, recurrence sites and survival rate were retrospectively analyzed and the appropriate extent of upward lymph node dissection for advanced lower rectal carcinoma was evaluated.

Classification of the Level of Upward Lymph Node Dissection

Standard surgical procedures at our institution were previously reported in detail $[11,12]$. The extent of upward lymph node dissection was classified as follows: UD1 is defined as resection of the mesorectum, UD2 as division of the root of the superior rectal artery with lymph node dissection below that level, UD3 as division of the root of the inferior mesenteric artery with lymph node dissection below that level and UD4 as UD3 with the addition of para-aortic lymph node dissection (fig. 1) [12]. The level of upward lymph node dissection was determined by preoperative and intraoperative findings. When a patient was diagnosed as stage I, UD1 to UD2 lymph node dissection was performed. UD2 to UD4 lymph node dissection was performed for patients with stage II or III tumor. UD4 was performed until the first half of the 1990s, but has not been performed thereafter because of excessive operative time, blood loss and a high incidence of postoperative sexual dysfunction, especially in males $[11,13,14]$.

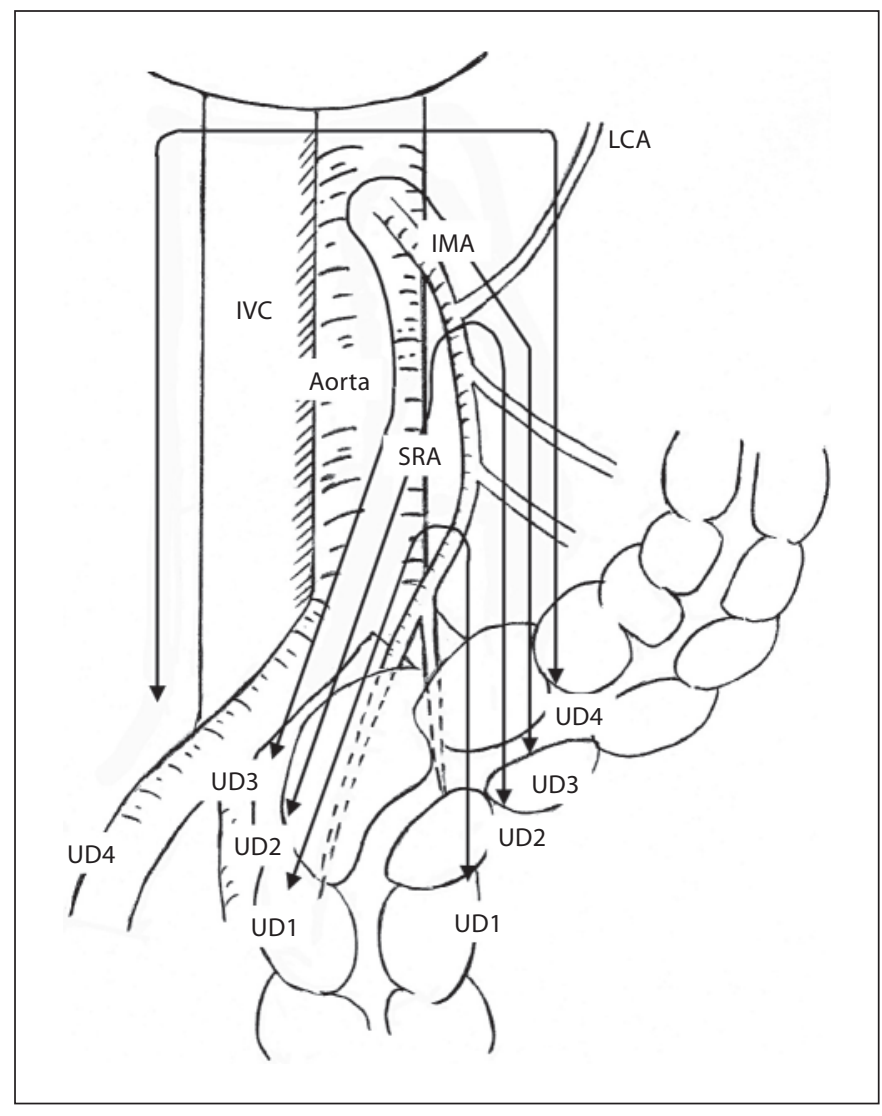

Fig. 1. Classification of the level of upward lymph node dissection. $\mathrm{UD} 1$ is defined as resection of the mesorectum; UD2 as division of the root of the superior rectal artery (SRA) and lymph node dissection below this level; UD3 as division of the root of the inferior mesenteric artery (IMA) and lymph node dissection below this level; and UD4 as UD3 with para-aortic lymph node dissection. IVC = Inferior vena cava; LCA = left colic artery.

\section{Statistical Analysis}

Survival curves were traced using the Kaplan-Meier method. The differences between curves were tested using the log-rank test. Comparisons between groups were performed using $\chi^{2}$ test. $\mathrm{p}<0.05$ was considered significant. All statistical calculations were made using SPSS computer software (SPSS 11.0, SPSS Inc., Chicago, Ill., USA).

\section{Results}

The characteristics of 285 patients according to the UD classification are shown in table 1 . There were 78 (27.4\%), 133 (46.7\%) and $74(26.0 \%)$ patients who underwent UD2, UD3 and UD4, respectively. All patients were followed up until death or for at least 3 years with a mean follow-up period of 66 months. The rate of sphincter-pre- 
Table 1. Patient characteristics according to the UD classification

\begin{tabular}{lclll}
\hline & Total $(\mathrm{n}=285)$ & UD2 $(\mathrm{n}=78)$ & UD3 $(\mathrm{n}=133)$ & UD4 (n=74) \\
\hline Age, years (mean) & 58.2 & 58.1 & 58.2 & 58.4 \\
Sex ratio (male:female) & $191: 94$ & $53: 25$ & $90: 43$ & $48: 26$ \\
Follow-up period (mean) & 66 & 59 & 57 & $88^{\mathrm{a}, \mathrm{c}}$ \\
Surgical procedure & & & & \\
$\quad$ Sphincter-preserving surgery & $143(50.2)$ & $53(67.9)$ & $64(48.1)$ & $26(35.1)^{\mathrm{a}, \mathrm{b}}$ \\
$\quad$ Non-sphincter-preserving surgery & $142(49.8)$ & $25(23.1)$ & $69(51.9)$ & $48(64.9)$ \\
Lateral LNs dissection & & & & $5(6.8)^{\mathrm{d}}$ \\
$\quad$ No & $68(23.9)$ & $32(41.0)$ & $31(23.3)$ & $69(93.2)$ \\
$\quad$ Yes & $417(76.1)$ & $46(59.0)$ & $102(76.7)$ & $57^{\mathrm{d}}$ \\
Evaluated LN, n (mean) & 3 & 31 & 39 & 3 \\
Metastatic LN, n (mean) & & 2 & $38(28.6)$ & $27(36.5)$ \\
TNM classification & $94(33.0)$ & $29(37.2)$ & $95(71.4)$ & $47(63.5)$ \\
$\quad$ Stage II & $191(67.0)$ & $49(62.8)$ & & \\
$\quad$ Stage III & & & & \\
\hline
\end{tabular}

Values in parentheses are percentages.

${ }^{\mathrm{a}} \mathrm{p}<0.05 \mathrm{UD} 2$ vs. UD3, ${ }^{\mathrm{b}} \mathrm{p}<0.05 \mathrm{UD} 2$ vs. UD4, ${ }^{\mathrm{c}} \mathrm{p}<0.05 \mathrm{UD} 3$ vs. UD4, ${ }^{\mathrm{d}} \mathrm{p}<0.05$ between each UD classification.

serving surgery was higher in UD2 patients than in those who underwent UD3 or UD4. The rate of undergoing lateral lymph node dissection and the number of evaluated lymph nodes increased significantly with the extension of upward lymph node dissection. However, there were no significant differences in the number of metastatic lymph nodes and the ratio of stage II to III among UD classifications.

In each TNM stage, the overall survival curves in relation to the extent of upward lymph node dissection were evaluated and there were no significant differences according to the extent of upward lymph node dissection (fig. 2). Recurrence sites after curative resection are demonstrated in table 2 . In both groups with or without lymph node dissection at the root of the inferior mesenteric artery, the lung was the most common site of recurrence followed by the liver. Recurrence sites did not significantly differ between the groups, including para-aortic or mediastinal lymph node metastases.

Table 3 summarizes the characteristics and outcomes of 4 patients with lymph node metastases at the root of the inferior mesenteric artery. They accounted for $1.9 \%$ of the 207 patients who underwent UD3 or UD4. Recurrences developed in all cases and their prognoses were significantly worse than those of the other stage III patients who underwent UD3 or UD4 ( $\mathrm{p}=0.011)$ (fig. 3). None of 4 patients survived for 5 years.
Table 2. Recurrent sites after curative resection

\begin{tabular}{lccc}
\hline Recurrent site & $\begin{array}{l}\text { UD2 } \\
(\mathrm{n}=78)\end{array}$ & $\begin{array}{l}\text { UD3/UD4 } \\
(\mathrm{n}=207)\end{array}$ & $\mathrm{p}$ value \\
\hline Lung & $16(20.5)$ & $36(17.4)$ & 0.543 \\
Liver & $6(7.7)$ & $19(9.2)$ & 0.692 \\
Pelvic cavity & $7(9.0)$ & $15(7.2)$ & 0.626 \\
Para-aortic or mediastinal LNs & $3(3.8)$ & $4(1.9)$ & 0.352 \\
\hline
\end{tabular}

Values in parentheses are percentages.

On the other hand, lymph node metastases along the superior rectal artery were discovered in 14 patients, excluding 3 patients with metastatic lymph nodes at the root of the inferior mesenteric artery, and table 4 shows their characteristics. They accounted for $4.9 \%$ of all patients. Ten patients developed recurrence and the lung was the most common site ( 6 patients), followed by the liver (2 patients). The 5-year overall survival rate was $61.2 \%$ in this group and there were no significant differences in overall survival among the patients with and without lymph node metastases along the superior rectal artery ( $\mathrm{p}=0.338)$ (fig. $4 \mathrm{a})$. In addition, there were no significant differences in survival of the patients with lymph node metastases along the superior rectal artery according to the extension of upward lymph node dissection performed (UD2 or UD3/4) ( $\mathrm{p}=0.642)$ (fig. 4b). 
Fig. 2. Overall survival curves in relation to the extent of upward lymph node dissection at each stage: (a) stage II and (b) stage III. There were no significant differences in each stage.

Fig. 3. Overall survival curves for the stage III patients with or without metastatic lymph nodes at the root of the inferior mesenteric artery (IMA). The former was significantly worse than the latter ( $\mathrm{p}=$ 0.011).
Stage II patients $(\mathrm{n}=94)$
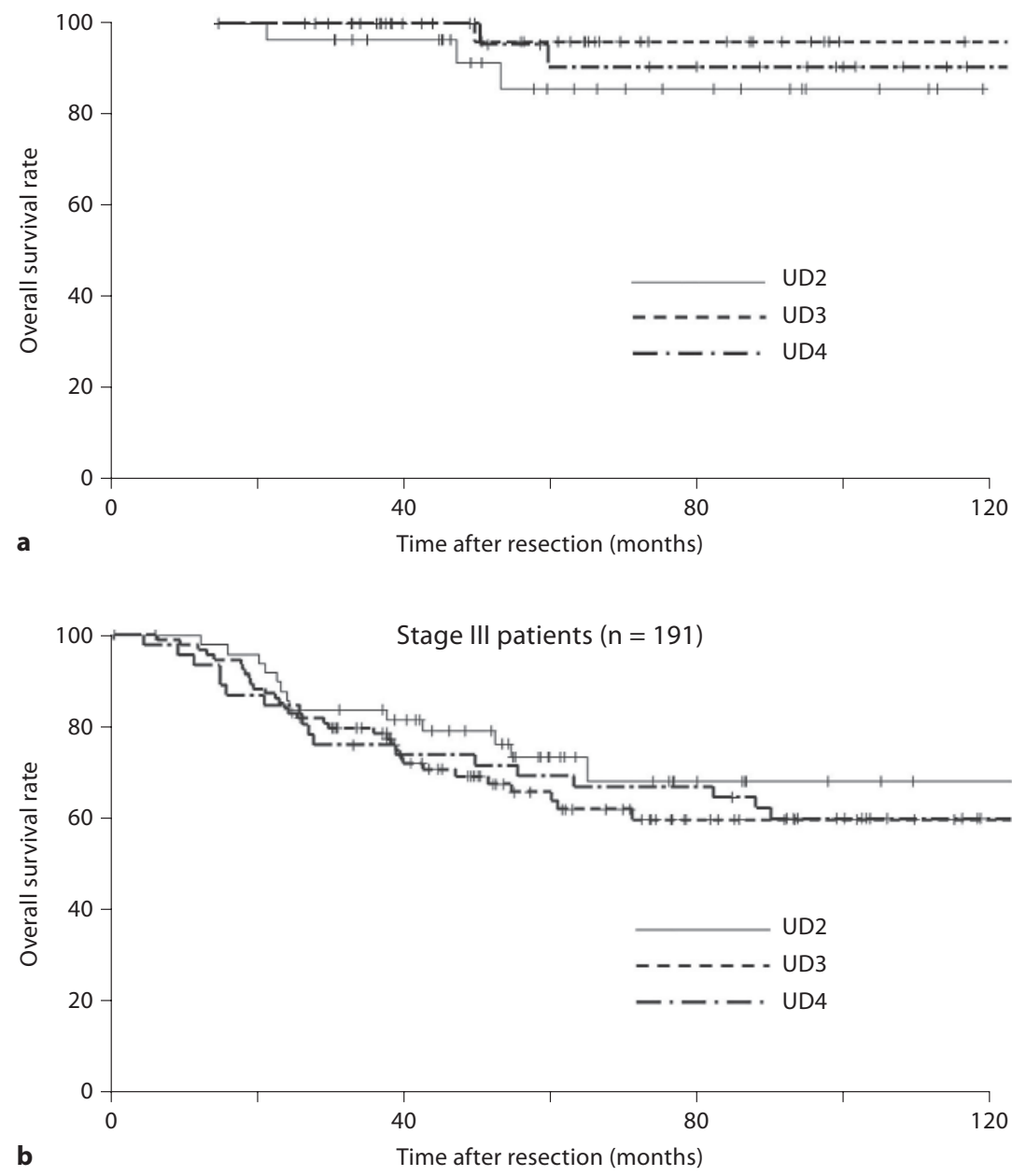

Stage III patients with UD3/4 $(n=142)$

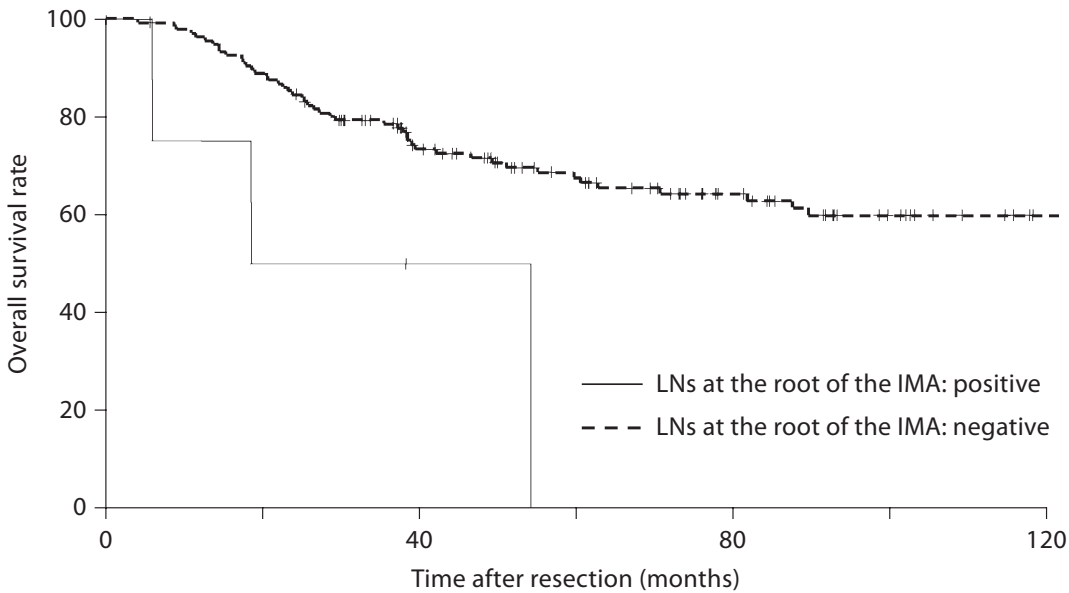


Table 3. Characteristics of the patients with metastatic LNs at the root of the inferior mesenteric artery

\begin{tabular}{|c|c|c|c|c|c|c|c|c|}
\hline Age & Sex & UD & Histology & $\mathrm{pT}$ & $\begin{array}{l}\text { Metastatic } \\
\text { LNs, n }\end{array}$ & $\begin{array}{l}\text { Recurrent } \\
\text { site }\end{array}$ & $\begin{array}{l}\text { Disease-free } \\
\text { time, months }\end{array}$ & $\begin{array}{l}\text { Outcome } \\
\text { months }\end{array}$ \\
\hline 33 & $\mathrm{~F}$ & 3 & well-differentiated adenocarcinoma & pT3 & 3 & lung, bone & 25 & died (54) \\
\hline 64 & $\mathrm{~F}$ & 3 & $\begin{array}{l}\text { moderately differentiated adeno- } \\
\text { carcinoma }\end{array}$ & pT3 & 4 & lung & 22 & $\begin{array}{l}\text { alive with recur- } \\
\text { rent tumor ( } 39)\end{array}$ \\
\hline 51 & M & 3 & poorly differentiated adenocarcinoma & pT3 & 25 & pelvic cavity & 11 & died (19) \\
\hline 57 & $\mathrm{M}$ & 3 & poorly differentiated adenocarcinoma & pT3 & 16 & $\begin{array}{l}\text { pelvic cavity, } \\
\text { peritonium }\end{array}$ & 4 & $\operatorname{died}(6)$ \\
\hline
\end{tabular}

\section{Discussion}

Surgical decisions regarding upward lymph node dissection for advanced lower rectal carcinoma remain controversial. In our study, patients with metastatic lymph nodes at the root of the inferior mesenteric artery comprised a small minority (4 patients, $1.9 \%$ ) and their prognoses were very poor. Their prognoses seemed to be almost equal to those of patients who underwent UD4 dissection and were pathologically proven to have metastatic para-aortic lymph node, although such patients are classified as stage IV in TNM classification and were excluded from this study. Furthermore, we could not demonstrate an effect of prophylactic lymph node dissection at the root of the inferior mesenteric artery in patients with any stage of disease. Moreover, lymph node dissection without the root of the inferior mesenteric artery did not result in increased para-aortic or mediastinal lymph node metastases, which we had thought might be caused by failing to perform lymph node dissection. We conclude that lymph node dissection at the root of the inferior mesenteric artery does not provide any survival advantage for patients with advanced lower rectal carcinoma and metastatic lymph nodes at this level have systematic disease.

Likewise, there were also a small number of patients with metastatic lymph nodes along the superior rectal artery (14 patients, $4.9 \%$ ) and the positive rate was far below the rate of lateral lymph nodes (55 of 217 patients who underwent lateral lymph node dissection, $25.3 \%$ ) in this series. However, the 5 -year overall survival rate in this group was $61.2 \%$ and there were no significant differences among stage III patients with and without lymph node metastases along the superior rectal artery. In addition, survival is no worse in patients with positive lymph node along the superior rectal artery as long as these positive nodes are resected by either UD2 or UD3/4. We conclude that UD2 lymph node dissection is adequate even for
Table 4. Characteristics of the patients with metastatic LNs along the SRA (exception for three with metastatic LNs at the root of the IMA)

$\begin{array}{llc}\text { Patients } & & 14 \\ \text { Age, years (mean) } & & 58.8 \\ \text { Sex ratio (male:female) } & & 12: 2 \\ \text { Upward LNs dissection } & \text { UD2 } & 4 \\ & \text { UD3 } & 6 \\ & \text { UD4 } & 4 \\ \text { Lateral LNs dissection } & \text { no } & 5 \\ & \text { unilateral pelvic } & 2 \\ \text { pT category in TNM } & \text { bilateral pelvic } & 7 \\ \text { classification } & \text { pT1 } & 2 \\ & \text { pT2 } & 2 \\ \text { pN category in TNM } & \text { pT3 } & 7 \\ \text { classification } & \text { pN1 } & 3 \\ \text { Recurrence } & \text { pN2 } & 7 \\ & \text { yes } & 7 \\ & \text { no } & 10 \\ & & 4\end{array}$

SRA = Superior rectal artery; IMA = inferior mesenteric artery.

stage III patients with lymph node metastases along the superior rectal artery.

There are some problems with the existing classifications of rectal carcinoma. TNM classification considers lymph nodes at the root of the inferior mesenteric artery as regional lymph nodes for colorectal carcinoma without regard to the location of the tumors, as well as lymph nodes along the superior rectal artery [10]. Under this classification, patients with metastatic regional lymph nodes are regarded as stage III and are subcategorized into three groups by the depth of tumor invasion and number of metastatic lymph nodes, not by the location of metastatic lymph nodes. The problem with this classification is that we cannot distinguish whether stage III patients have lymph node metastases at the root of the inferior mesenteric artery. 
Fig. 4. a Overall survival curves for stage III patients with or without metastatic lymph nodes along the superior rectal artery, excluding 4 patients with lymph node metastases at the root of the inferior mesenteric artery. There were no significant differences in overall survival between both groups $(\mathrm{p}=0.338)$. b Overall survival curves in relation to the extent of upward lymph node dissection for stage III patients with metastatic lymph nodes along the superior rectal artery, excluding 3 patients with lymph node metastases at the root of the inferior mesenteric artery. There were no significant differences in survival of the patients with lymph node metastases along the superior rectal artery according to the extension of upward lymph node dissection performed (UD2 or $\mathrm{UD} 3 / 4)(\mathrm{p}=0.642)$.

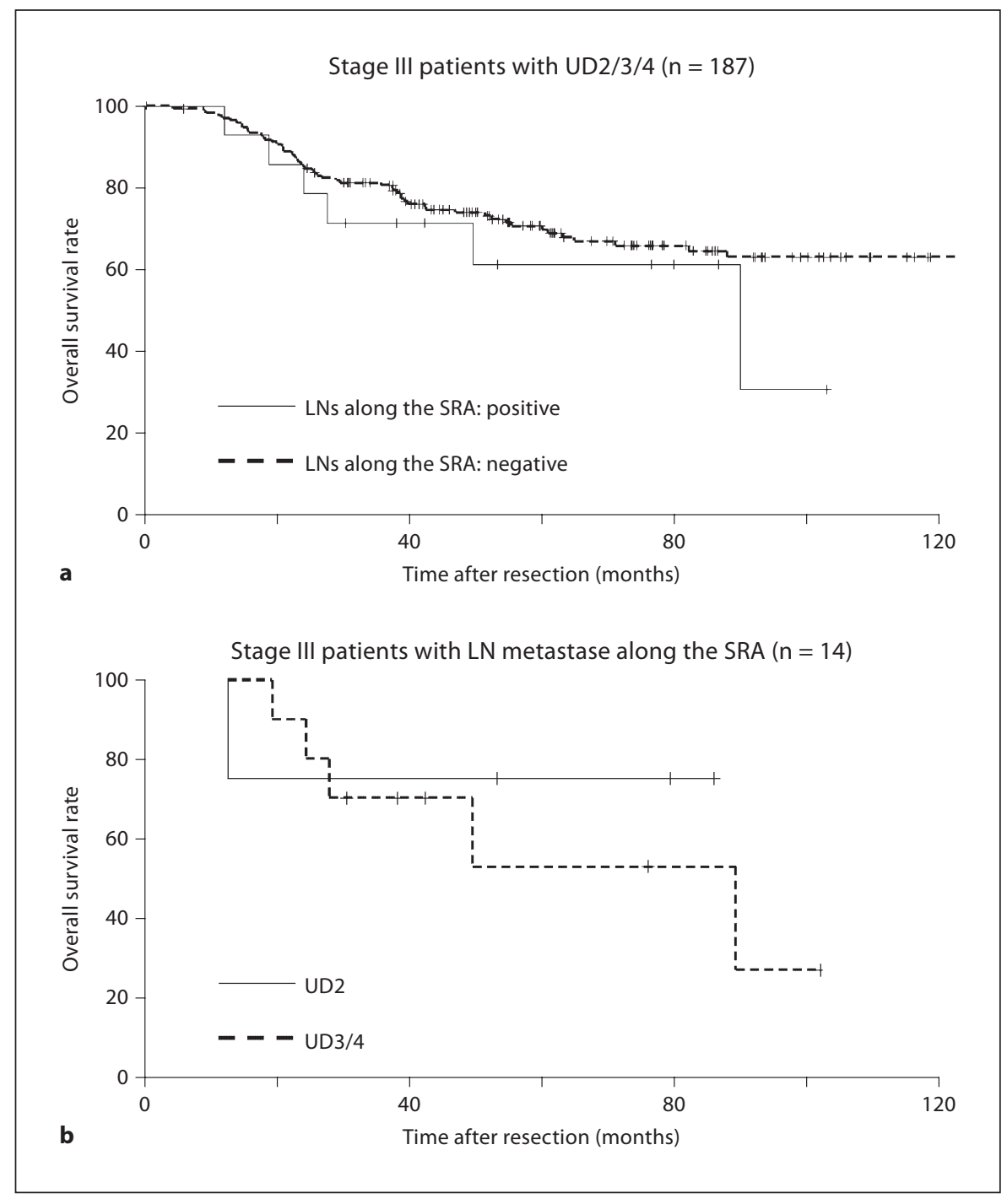

In comparison, the Japanese classification of colorectal carcinoma [9] treats regional lymph nodes in rectal carcinoma as follows: pararectal lymph nodes are defined as group 1, lymph nodes along the superior rectal artery as intermediate lymph nodes (group 2) and lymph nodes at the root of the inferior mesenteric artery as the main lymph nodes (group 3). However, this classification defines patients with metastatic lymph nodes in group 2 and/or group 3 as same stage (stage IIIb). Based on the results of this study, these criteria should be reevaluated.

In recent years, sphincter-preserving surgery has been increasingly adopted in patients with lower rectal carcinoma $[15,16]$. The most important postoperative complication in this procedure is anastomotic leakage. To avoid this complication, all colorectal surgeons pay attention to blood flow in the remnant colon, together with the tension of the anastomosis. Therefore, Western surgeons perform mobilization of the splenic flexure for most patients [17], but the position of the splenic flexure in Japanese is usually very deep in the left upper subphrenic area and it is sometimes rather difficult to mobilize the left side colon. However, Japanese patients usually have a long sigmoid colon, and if the surgeon preserves 1 or 2 arcades of marginal vessels of the sigmoid colon by dividing the sigmoid artery between the superior rectal artery and these marginal vessels, mobilization of the splenic flexure becomes unnecessary. In this situation, arterial blood flow is not being compensated. Preservation of the blood flow of the left colic artery is one solution to this problem, 
because the appropriate extent of upward lymph node dissection for lower rectal carcinoma is considered to be UD2. When the length of the vascular pedicle for lower anastomosis is short, we can cut the periphery of the left colic artery. Some surgeons choose left colic artery-preserving lymph node dissection at the root of the inferior mesenteric artery, but this increases the risk of damaging the lumbar splanchnic nerve.

Another problem encountered with lymph node dissection for lower rectal surgery is lateral lymph node dissection. Some reports mainly from Japan have supported the effectiveness of lateral pelvic lymph node dissection, and it is well established as the standard procedure in leading hospitals in Japan. However, in Western countries, the survival benefits of lateral pelvic lymph node dissection are regarded as doubtful. Instead, preoperative chemoradiotherapy is widely performed $[18,19]$. To resolve this disparity, a multicentric randomized clinical trial that compares lateral pelvic lymph node dissection with autonomic nerve preservation to total mesenteric excision (JCOG0212) is underway in Japan and data regarding this issue will become available in the near future [20].

In conclusion, survival of a minority with metastatic lymph nodes at the root of the inferior mesenteric artery was very poor. In addition, survival is no worse in patients with positive lymph node along the superior rectal artery as long as these positive nodes are resected by either UD2 or UD3/4. Surgeons should take these data into consideration and recognize that low ligation is adequate for advanced lower rectal carcinoma.

\section{References}

1 Moriya Y, Sugihara K, Akasu T, Fujita S: Nerve-sparing surgery with lateral node dissection for advanced lower rectal cancer. Eur J Cancer 1995;31A:1229-1232.

2 Moriya Y, Sugihara K, Akasu T, Fujita S: Importance of extended lymphadenectomy with lateral node dissection for advanced lower rectal cancer. World J Surg 1997;21: 728-732.

- 3 Fujita S, Yamamoto S, Akasu T, Moriya Y: Lateral pelvic lymph node dissection for advanced lower rectal cancer. Br J Surg 2003; 90:1580-1585.

4 Ueno M, Oya M, Azekura K, Yamaguchi T, Muto T: Incidence and prognostic significance of lateral lymph node metastasis in patients with advanced low rectal cancer. $\mathrm{Br} \mathrm{J}$ Surg 2005;92:756-763.

5 Shida H, Ban K, Matsumoto M, Masuda K, Imanari T, Machida T, Yamamoto T: Prognostic significance of location of lymph node metastases in colorectal cancer. Dis Colon Rectum 1992;35:1046-1050.

-6 Surtees P, Ritchie JK, Phillips RK: High versus low ligation of the inferior mesenteric artery in rectal cancer. Br J Surg 1990;77:618621.

7 Slanetz CA Jr, Grimson R: Effect of high and intermediate ligation on survival and recurrence rates following curative resection of colorectal cancer. Dis Colon Rectum 1997; 40:1205-1218.
8 Pezim ME, Nicholls RJ: Survival after high or low ligation of the inferior mesenteric artery during curative surgery for rectal cancer. Ann Surg 1984;200:729-733.

9 Japanese Society for Cancer of the Colon and Rectum: Japanese Classification of Colorectal Carcinoma, ed 1. Tokyo, Kanehara, 1997.

10 International Union against Cancer: TNM classification of malignant tumours, ed 6 . New York, Wiley-Liss, 2002.

11 Moriya Y: Pelvic node dissection with autonomic nerve sparing for invasive lower rectal cancer: Japanese experience; in Wanebo HJ (ed): Colorectal Cancer. St. Louis, Mosby, 1993, pp 274-289.

12 Akasu T, Moriya Y: Abdominopelvic lymphadenectomy with autonomic nerve preservation for carcinoma of the rectum: Japanese experience; in Wanebo HJ (ed): Surgery for Gastrointestinal Cancer: A Multidisciplinary Approach. Philadelphia, LippincottRaven, 1997, pp 667-680.

$\checkmark 13$ Maas CP, Moriya Y, Steup WH, Kiebert GM, Kranenbarg WM, van de Velde CJ: Radical and nerve-preserving surgery for rectal cancer in The Netherlands: a prospective study on morbidity and functional outcome. $\mathrm{Br} \mathrm{J}$ Surg 1998;85:92-97.

14 Sugihara K, Moriya Y, Akasu T, Fujita S: Pelvic autonomic nerve preservation for patients with rectal carcinoma: oncologic and functional outcome. Cancer 1996;78:18711880.
5 Schiessel R, Novi G, Holzer B, Rosen HR, Renner K, Holbling N, Feil W, Urban M: Technique and long-term results of intersphincteric resection for low rectal cancer. Dis Colon Rectum 2005;48:1858-1867.

16 Rullier E, Laurent C, Bretagnol F, Rullier A, Vendrely V, Zerbib F: Sphincter-saving resection for all rectal carcinomas: the end of the 2-cm distal rule. Ann Surg 2005;241: 465-469.

17 Milson JW, Stolfi VM: Low rectal and midrectal cancers; in Wanebo HJ (ed): Colorectal Cancer. St. Louis, Mosby, 1993, pp 214-241.

18 Rullier E, Goffre B, Bonnel C, Zerbib F, Caudry M, Saric J: Preoperative radiochemotherapy and sphincter-saving resection for T3 carcinomas of the lower third of the rectum. Ann Surg 2001;234:633-640.

19 Schaffer M, Thoma M, Wilkowski R, Schaffer P, Duhmke E: Radio-chemotherapy as a preoperative treatment for advanced rectal cancer: evaluation of down-staging and morbidity. Onkologie 2002;25:352-356.

20 http://clinicaltrials.gov/ct/gui/show/ NCT00190541 [ClinicalTrials.gov web site]. Accessed October 4, 2006. 